\title{
PULMONARY ARTERY THROMBOSIS FOLLOWING CARDIAC CATHETERIZATION
}

\author{
BY \\ J. A. NIGHTINGALE AND B. L. WILLIAMS \\ From the Departments of Cardiology and Pathology, University of Manchester
}

Major thrombosis of a primary division of the pulmonary artery does not appear to have been recorded as a complication of cardiac catheterization. It therefore seems desirable to record the following case of mitral stenosis in which thrombosis of the right main branch of the pulmonary artery followed catheterization.

\section{Case Report}

A 33-year-old housewife was admitted to the Manchester Royal Infirmary under the care of Professor Bramwell, suffering from chronic rheumatic heart disease with mitral stenosis, auricular fibrillation, and congestive heart failure which responded to medical treatment. Cardiac catheterization was performed four weeks after admission. On arrival in the theatre the patient had a rapid auricular fibrillation but her general condition was satisfactory. A No. 8 catheter introduced into the left median cubital vein passed easily to the right main branch of the pulmonary artery where the resting mean pressure was over $78 \mathrm{~mm}$. of mercury. The catheter was then advanced to the wedge position in the right mid-zone where a mean pressure of $24 \mathrm{~mm}$. mercury was recorded. Due to technical difficulty with the saline manometer, it remained in this position for 29 minutes, apart from a short period of withdrawal into the pulmonary artery to test the catheter's patency. Pressure readings and blood samples were taken during withdrawal. Throughout the procedure a slow drip of $\frac{1}{2}$ per cent procaine saline containing 46,000 units heparin/litre was given. All manipulation was under radioscopic control. Five hours after catheterization the patient became dyspnœic and cyanosed, the tachycardia persisting. Following treatment she regained her usual state within 36 hours and her condition remained unchanged until mitral valvotomy was performed three weeks after catheterization. Splitting of the commissure presented no difficulty, but spontaneous respiration was not re-established and the patient died six hours after operation.

\section{Autopsy}

The heart weighed $450 \mathrm{~g}$., the right ventricle being dilated and hypertrophied and the left auricle dilated. The pericardial sac contained a few ml. of blood-stained fluid. The cusps of the mitral valve were opaque and thickened and the chordæ tendineæ shortened. The anterolateral commissure had been split so that the valve admitted two fingers. The tricuspid orifice was dilated, admitting five fingers; the valve cusps were healthy. The pulmonary and aortic valves were normal. In the right branch of the pulmonary artery was a firm ante-mortem thrombus, $8 \mathrm{~cm}$. in length and $2 \mathrm{~cm}$. in diameter (Fig. 1). The thrombus had a firm linear attachment, $4 \mathrm{~mm}$. wide, to the posterior wall of the pulmonary artery for a distance of $6 \mathrm{~cm}$; the proximal $2 \mathrm{~cm}$. of the thrombus was free in the lumen, projecting into the main pulmonary trunk at its bifurcation. The lumen of the artery was patent, though much reduced in this region. The intima was moderately atheromatous. The lungs were congested and odematous. The left lung was partially collapsed and the left pleural sac contained a little blood-stained fluid. No infarcts were present and there was no thrombosis of the smaller arteries. Chronic venous congestion was present in the liver, spleen, and kidneys. The other organs were normal.

\section{Histology}

Sections taken through the thrombus and pulmonary artery (Fig. 2) showed the thrombus firmly united to the thickened intima of the artery; there were many dilated capillaries in the adventitia of the vessel. The thrombus contained many fibroblasts and young capillaries, penetrating to a depth of $2 \mathrm{~mm}$., and judged by the degree of organization appeared to be about 3 weeks old. 


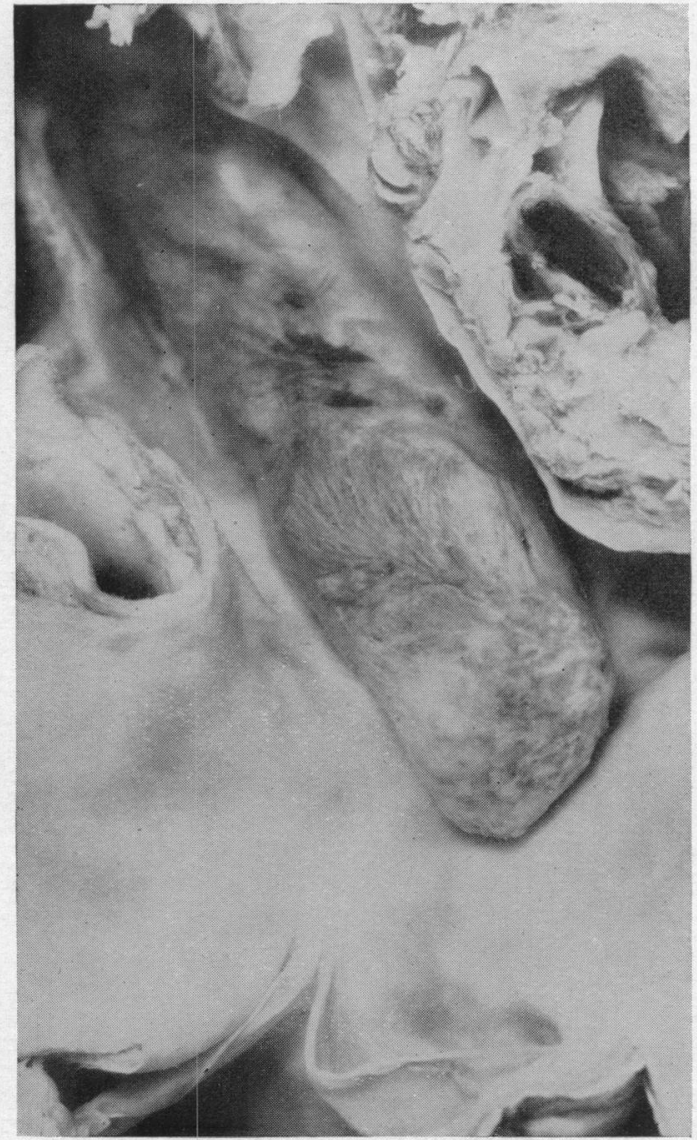

Fig. 1.-Thrombus in the right main branch of the pulmonary artery.

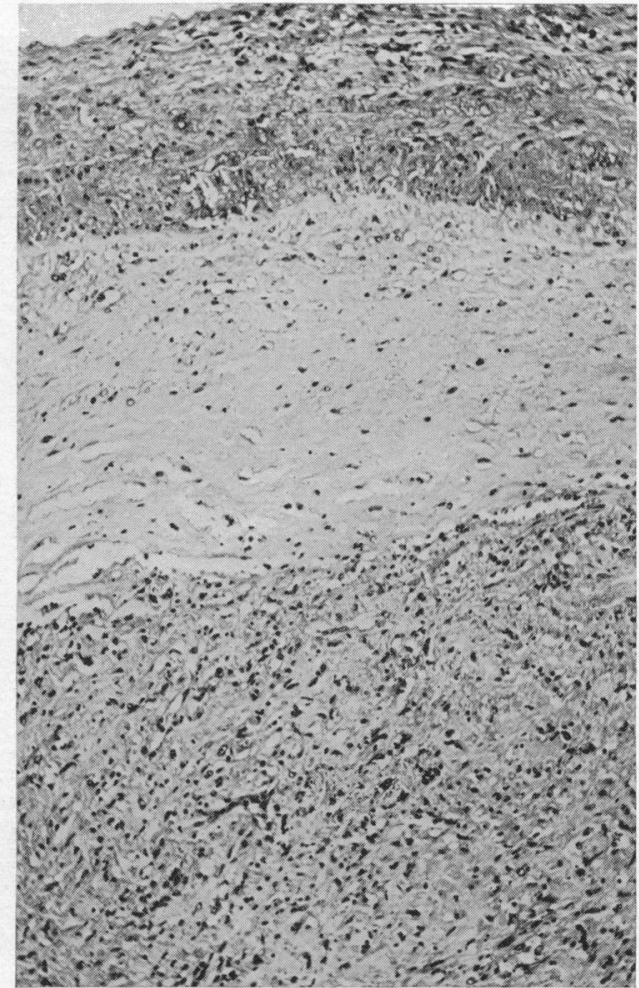

FIG. 2.-Photomicrograph showing the organizing thrombus and the thickened intima of the pulmonary artery.

\section{Discussion}

The evidence suggests that thrombosis of the right main branch of the pulmonary artery was the sequel of cardiac catheterization. Coincidental spontaneous thrombus formation (Billings, 1921; Brenner, 1935) is a possibility, but the site and shape of the thrombus, its age, and the demonstration of patency of the right pulmonary artery at the time of catheterization are against this explanation. Experimentally, endocardial lesions have been found following catheterization in dogs (Ellis et al., 1950; Goodale et al., 1947), but similar lesions in the pulmonary artery do not appear to have been described. Goodwin (1953) records a fatal case of subendocardial hæmorrhage in the right ventricle and postulates the occurrence of similar non-fatal lesions in other cases. Edwards et al. (1953) report the case of a patient dying 24 hours after catheterization from cardiac failure secondary to mitral stenosis, who showed a fibrinous deposit in the main stem of the pulmonary artery, probably overlying an endothelial erosion; this may represent an earlier stage of the lesion described in the present case. In mitral stenosis the atheromatous state of the pulmonary artery and its branches would predispose to minor endothelial damage by a catheter or its tip.

Although conditions are not strictly comparable, it seemed worth while to catheterize cadavers in order to discover the possible sites of damage to the pulmonary artery during cardiac catheterization. In passing the catheter to the wedge pressure position considerable buckling occurs, and, according to the position of the terminal artery catheterized, the buckled catheter may press on the 
superior, posterior, or anterior wall of the main stem pulmonary artery. The catheter tip has also been seen to impinge on the posterior wall during manipulation. Similar buckling of the catheter is often observed radioscopically during the actual recording of the wedge pressure. Either of these mechanisms or unusually prolonged contact may, in the case described, have initiated thrombosis in an atheromatous artery. The risk of pulmonary infarction following the wedge pressure technique is well recognized (Miller et al., 1952; Houssey et al., 1952) but this is peripheral in type and not due to thrombosis of a large pulmonary artery.

It is worth emphasizing that in our case the thrombosis was symptomless in life and but for the patient's post-operative death might have passed unrecognized. To prevent the occurrence of similar lesions it would be advisable, after insertion into the wedge position, to withdraw the catheter as far as possible without dislodging the tip. This manœuvre reduces buckling of the catheter and lessens the risk of trauma in the region of the pulmonary artery main stem. The time spent in the wedge position should be as short as possible.

\section{SUMMARY}

An instance of thrombosis of the right main branch of the pulmonary artery following cardiac catheterization is described. Possible mechanisms of production are discussed.

We wish to thank Professor Crighton Bramwell and Professor A. C. P. Campbell for help in the preparation of this paper.

\section{REFERENCES}

Billings, F. T. (1921). Pennsylvania Med. J., 25, 152.

Brenner, O. (1935). Arch. intern. Med., 56, 211, 457, 724, 976, 1189.

Edwards, J. E., Helmholz, H. F., Du Shane, J. W., and Burchell, H. B. (1953). Proc. Mayo Clin., $28,113$.

Ellis, E. J., Essex, H. E., and Edwards, J. E. (1950). Proc. Mayo Clin., 25, 73.

Goodale, W. T., Lubin, M., Eckenhoff, J. E., Hafkenschiel, J. H., Durlacher, S. H., Landing, B. H., and Banfield, W. G. (1947). Proc. Soc. Exper. Biol. Med., 66, 571.

Goodwin, J. F. (1953). Brit. Heart J., 15, 330.

Houssey, H. E. J., Haynes, F. W., Dexter, L. (1952). Proc. Soc. Exper. Biol. Med., 79, 444.

Miller, G., Goldberg, H., Elisberg, E. I., Snider, G. L., Toor, M., and Katz, L. N. (1952). J. Lab. Clin. Med., 40, 390. 